\title{
A Toolkit for Animal Touchscreen Slider Design
}

\author{
Jacob Logas \\ logasja@gatech.edu \\ Lorita Freeman \\ lorita.freeman@gatech.edu
}

\author{
Will Mitchell \\ wmitchell30@gatech.edu \\ Clint Zeagler \\ clintzeagler@gatech.edu \\ Georgia Institute of Technology \\ Atlanta, Georgia
}

\author{
Monira Khan \\ monirakhan@gatech.edu \\ Melody Moore Jackson \\ melodymoorejackson@gatech.edu
}

\begin{abstract}
Animal-computer interaction is a quickly growing field in computer science however unlike HCI, there are no tools to ease interface development for its users, increasing overhead. Here, we present the beginning of a toolkit created specifically for animal interactions. We implement a gesturebased interface, a slider, as it is highly configurable in shape, colour, and feedback allowing it to be as simple or complex as desired. The resultant interface can be in any shape, provides 20 run-time configuration options, and collects usage data for qualitative analysis. The toolkit reduces the effort needed to build gestural animal interfaces for research or everyday use.
\end{abstract}

\section{CCS CONCEPTS}

\section{- Human-centered computing $\rightarrow$ Touch screens; User} interface toolkits;

\section{KEYWORDS}

Touchscreen; Toolkit; Virtual User Interface; Animal Computer Interaction

\section{INTRODUCTION}

An increasing subset of the animal kingdom is being evaluated in interaction studies as the Animal-Computer Interaction (ACI) field grows. However, existing interface design tools are understandably designed for human interaction

Permission to make digital or hard copies of all or part of this work for personal or classroom use is granted without fee provided that copies are not made or distributed for profit or commercial advantage and that copies bear this notice and the full citation on the first page. Copyrights for components of this work owned by others than the author(s) must be honored. Abstracting with credit is permitted. To copy otherwise, or republish, to post on servers or to redistribute to lists, requires prior specific permission and/or a fee. Request permissions from permissions@acm.org.

ACI18, December 4-6, 2018, Atlanta, GA, USA

(c) 2018 Copyright held by the owner/author(s). Publication rights licensed to ACM.

ACM ISBN 978-1-4503-6219-1/18/12 ..\$15.00

https://doi.org/10.1145/3295598.3295611 imposing hard constraints on interfaces based on HumanComputer Interaction (HCI) research [13]. Thus when making an animal interface, designers must choose to either heavily modify existing toolkits or build interfaces without aid. Animal interfaces have the added difficulty of accurately quantifying interface efficacy without being able to survey participants. These added complexities cause interface and interaction researchers to eschew complex interactions and as a result, most animal interfaces have simple single-touch interactions $[1,4,8,11,12,15,16]$. Here, we hope to provide a tool that is able to enhance a designer's ability to create innovative interactions and automatically collect quantifiable metrics of the interaction.

Although the interactions in them are simple, recent ACI studies expose important design considerations for animalcentric interfaces. First, animal eye physiology affects interface experience as the visible colour spectrum often differs from that of humans. This disparity between human and animal vision can cause seemingly separable elements to humans indistinguishable to animal participants. In effect, a designer must design as if they are creating for the colourblind. Second, interface designers need to be cognizant of how the animal naturally interacts with the world. In touchscreen studies, animals often use their snouts to interact causing them to be close to the screen. This reality invalidates many human design principles that assume the user is at a comfortable viewing distance. Finally, designers need to account for the varying size of animals that will use the interface. In humans, this is not so much of an issue as overall designers can expect most ages to be dexterous with their digits when using touchscreens. However, with animals, age and breed can cause the scale of an interface to vary wildly between species participants as the interface elements all need to be reachable by the animal's snout. These considerations were all discovered by interface evaluations made possible by careful observation and analysis of collected metric data.

Interface evaluations are necessary to quantitatively measure the experience and efficacy of an interface. Animal studies cannot use qualitative measures that HCI studies use, 
but methods for indirect evaluation from HCI can be modified for use with animals [5]. In both HCI and ACI it is still an open question as to what the optimal set of metrics are for sufficiently quantifying interfaces and it is unlikely that they will be the same across species. Advanced metrics like gaze tracking or user pose analysis may provide greater insight [9], but thorough analysis of touch and temporal metrics seem like a good predictor of experience.

Wigdor and Wixon describe in their text the primitives of interfaces and how they can differ between hardware [13]. We propose that not only do interface primitives differ between hardware, but also between species. In ACI, single touch interactions are regularly used likely because the difference between the human and animal interface is minimal and thus easier to identify optimization for animals. Gestural interfaces, on the other hand, require a more nuanced look at what gestures are natural for an animal to make, and it is unclear if the preference is at all the same as humans. These kinds of studies require many iterations, which when hampered by constrained design tools require more resources. Thus, a slider interaction is chosen for the pilot interface primitive in an effort to accelerate animal gesture studies.

Compared to discrete interactions like buttons, a gestural interaction can allow for more informative and natural interfaces. Creating an animal-centric gesture interface is difficult though because traditional design tools constrain gesture inputs to those known to work well for humans. These constraints make designing a good human interface easier but hamper efforts to create innovative interfaces for nonhumans. When we relax the constraints, the slider's large number of configuration axes are revealed. This high dimension of configurability increases the space of possible interactions well beyond that of other elements and makes gesture studies easy to iterate on.

The major contributions of this paper are as follows:

(1) Present a toolkit made for animal interaction design

(2) Implement an interaction that is largely unexplored

(3) Illustrate usefulness with use cases

\section{RELATED WORK}

In the field of $\mathrm{HCI}$, much research has been done into how interactions can be made more intuitive to the user [2, 3]. Intuitive use of products here is defined by Blacker and Hurtienne as using products utilizing knowledge gained through other experiences. This, they argue, means that intuitive interfaces need features the user has encountered before. This makes it especially difficult to design for animals as the human and animal experience differ greatly. Thus, following standard practices in interface design likely creates an interface intuitive to the human designer but difficult for animals to understand. This is why in ACI there is an effort to discover how to include the participant animals into the design [6]. An animal's inability to intuitively use existing human interfaces likely led to the belief that animals are cognitively incapable of using an interface. However, cognition studies have refuted this belief by illustrating the reasoning skills of species across the animal kingdom, often with digital interfaces.

An important cognitive ability necessary for intuitive interaction with interfaces is to reason by inference, meaning the user is able to make an inference based on past experience. Aust et al. investigated this in dogs and pigeons by training them on a subset of stimuli as either providing a positive or negative reward. Then three stimuli were presented with two previously trained as negative and one unknown. Approximately half the dogs chose the unknown when presented with two known negatives, but were less likely to choose unknown with a positive present. Pigeons on the other hand overwhelmingly chose the known stimuli, likely indicating a neophobic nature. The results indicate dogs have the ability to reason by exclusion while pigeons do not [1]. Though, studies show other bird species do have exclusionary reasoning skills [7]. The results here indicate that not only do some animals have the prerequisite cognition to use interfaces intuitively but also that the range of abilities within a genus can widely vary.

Another necessary cognitive skill for intuitive use of digital interfaces is the transfer of concepts. Interfaces are much easier to approach if it represents a concept that the user already knows. Range et al. evaluated domestic dogs' ability to transfer their concept of a dog to a $2 \mathrm{D}$ interface. The study presented images of dogs and landscapes which the participant was meant to classify whether or not a dog is present [10]. Vonk et al. similarly studies concept formation in American black bears (Ursus americanus) [11]. Both studies indicate that some animals have requisite ability to use interfaces intelligently as opposed to just replicating a taught behaviour.

Digital animal interfaces are a good tool for cognition studies but, as with humans, they can be used to enrich the animal's life. Perdue et al. designed a touchscreen enrichment tool for orangutans in a zoo exhibit. Being that orangutans have digits like humans, the interface required little changes of consideration from human consideration. It provided the animals with problem-solving tasks that when done correctly dispensed treats [8]. This interface did not have any gestural inputs but instead relied on single-touch interactions. Other animals were not as simple to design for. Zeagler et al. designed an emergency alert system to be operated by a service dog while at home. They identified several considerations when designing an interface for dogs. First, larger buttons are preferable as the dog is interacting 
with its snout, making its touch points less accurate. Second, some shades of colour visible to dogs were too close in their vision to be distinguishable. Lastly, the animals using the system naturally began to swipe across the sequential threebutton interface as it resulted in a faster reward [15]. This last consideration was another motivation to implement a gestural interface as they appear to be intuitive to dogs.

\section{TOUCHSCREEN SLIDER INTERACTIONS}

Normally touchscreen gestures are free-form, requiring the user to complete it in a single touch and memorize the shape. A modified slider interface can relax these constraints by adding a specific touch-point (handle), a visible and well-defined path, and the ability for the gesture to be completed in multiple touches. Here, we aim to aid the exploration of animal abilities in moving visual objects along defined pathways. This, we hope, will result in the discovery of innovative interfaces natural to the target animal.

\section{Concept Formation Testing}

As stated before, there have been several studies into certain species' ability to form and use concepts on digital interfaces $[10,11]$. Though in these cases the testing interfaces needed to be built from the bottom up and only tested on simple interfaces.

A study of interface element concept formation can be carried out using a slider interface of varying shape. The sliders for this case increase in complexity with the progression of shape demonstrated in Figure 1. First, the animal would be trained on a linear slider such as the first image in the figure. Second, a U-Shaped slider is introduced as an intermediate step, challenging the animal to apply the slider concept to an unfamiliar shape but still being guided through the interaction. Finally, an S-Shaped slider is evaluated without training. Evaluation of the animal would be carried out by analysis of the time to completion and gesture accuracy compared to the slider path. This is a rudimentary difficulty progression design and can have any number of steps added in between.

\section{Aesthetic Design Effect}

Designs in human interfaces are made to encourage interaction or suggest how an interface is used. It is unclear whether animals respond to this kind of design. This hypothesis could be tested using a slider interface by training the animal on a directional design and then evaluating how the animal responds to a violation of the design.

A triangle-shaped slider handle can suggest a direction of interaction. Creating a set of slider interfaces with directional handles to train animal participants on and then evaluating performance on sliders violating the directionality can aid in determining responsiveness to suggestive design. These interfaces need largely the same metrics as before to quantitatively analyze the effect of violating design. For example, after changing directionality the animal's gestures can be evaluated to determine if they are in the suggested direction or correctly along the slider path.

\section{Feedback Evaluation}

Exploring the efficacy of different feedback schemes is of great importance to ACI. It is still an open question as to what feedback animals prefer. It is even unclear if there is any overlap in feedback optimality between species. Thus for each animal species, a study of feedback schemes must be carried out. Here, we describe a simple feedback scheme. It interpolates the colour of various parts of the scene based on the progression of a slider. This scheme will identify if the participant responds to colour-based visual stimuli. Efficacy of this scheme can be quantifiably measured by the time to completion of the task and number of iterations needed to learn the interaction. This same approach can be easily applied to other feedback methods such as tactile motors or sound modulation.

\section{SLIDER TOOLKIT}

We created this toolkit to enable rapid prototyping and evaluation of slider interfaces with various shapes, colour pallets, and feedback schemes. A configurable shape is available to allow designers to create gestural interfaces that range from simple to complex. A modifiable colour pallet is important as it allows the toolkit to be used with any animal participant regardless of their colour vision. Finally, the feedback mechanism is exposed to a designer to allow quick prototyping and evaluation of feedback schemes.

\section{Slider}

A slider is made up of two major parts, the handle and the track. The handle is the main interaction point that the user selects and drags along the track. The track is the path the 
handle must follow and determines the complexity of the interaction.

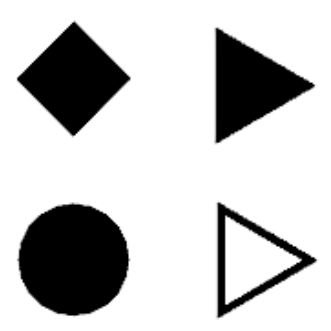

Figure 2: Possible handle configurations. erated using equidistant points on a circle of radius $r$. Figure 2 illustrates some of the handle shapes possible with this implementation. The handle also has an outline component allowing multi-coloured handles as well as "hollow" handles, shapes with a transparent centre. The handle can be given physicality, which allows phys-
The handle's shape is genical forces like gravity or momentum to manipulate it. We

believe that this will increase interest for animals by making the interface more closely represent a real-world object.

The slider track is implemented using Bezier curves. These are created using an arbitrary number of user-defined anchor points with tangents for each. The Bezier algorithm then solves for the equation of the line between the two points that will end with the given tangents. With the equation, the line is rendered by sampling points along the line at a given resolution. In the toolkit, designers define the anchor points, tangents of the points, and resolution of the track allowing nearly limitless configurability. Figure 3 illustrates curve configuration.

In the toolkit, when the curve is being drawn linear colliders are placed along the outside of the slider. The distance of these "bumpers" from the centre of the track is configurable, allowing varying degrees of path stringency. This approach is taken in place of a strictly track-based interaction as some animals cannot use interfaces as dexterously as humans. Thus they are not physically able to precisely follow the track.
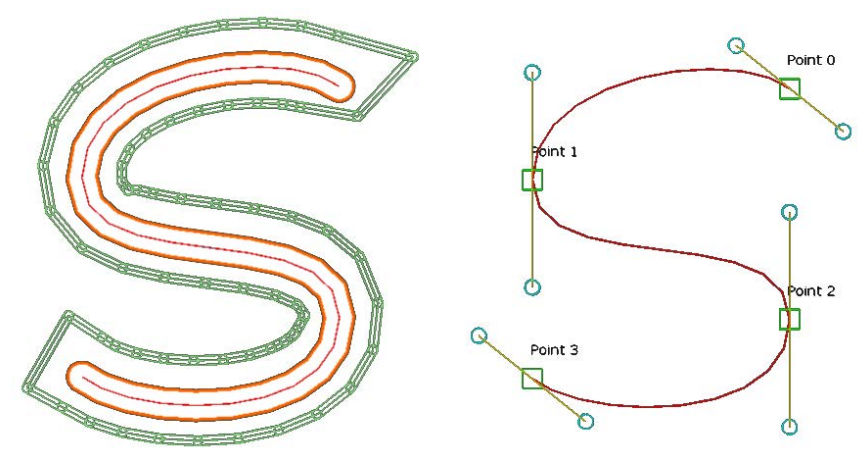

Figure 3: Slider shape configuration using Bezier curves and the resulting "bumpers".

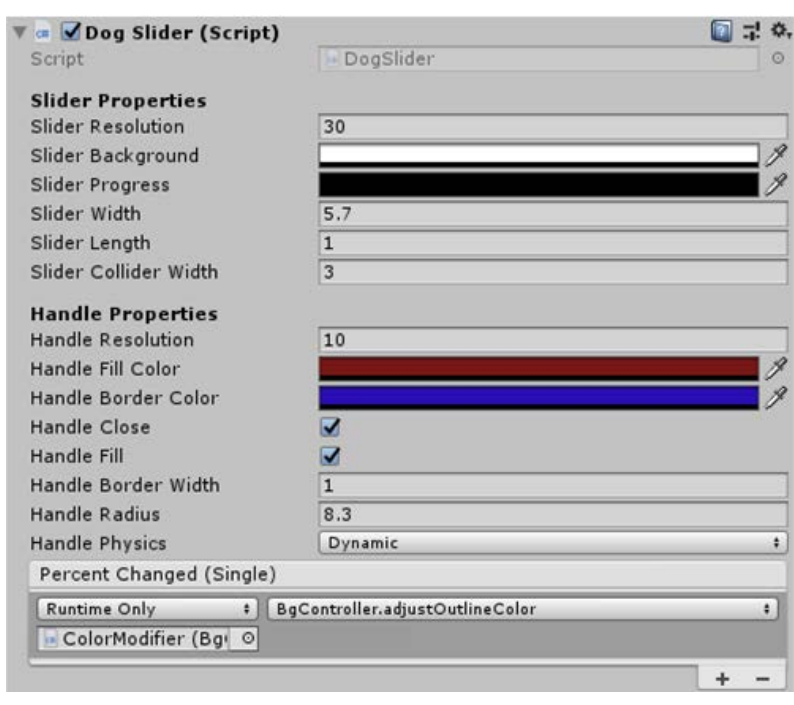

Figure 5: Offline configuration menu in Unity editor.

\section{Configuration Menus}

The toolkit provides two main interfaces by which the slider can be configured. One displayable during run-time and one inside the Unity editor. The in-game configuration allows for quick changes to the interface during run-time but with more basic, mostly cosmetic, options. The configuration menu in the Unity editor, on the other hand, provides more advanced options but requires the program to be stopped and rebuilt.

The first configuration menu presents itself as a tri-bar menu, activated using the button at the top left of the interface. It allows for quick cosmetic modifications and variations to be tested. Figure 4 shows the interface and the actions available to the user. Even though it does not provide advanced control over the interface, it allows researchers to determine an optimal cosmetic configuration quickly.

The second configuration menu, as seen in Figure 5, exposes top-level aspects of the slider and handle properties. This allows the designer to have advanced control over the interface. Here, the designer can determine several cosmetic and functional aspects such as the stringency of the track path, physical attributes of the handle, and the feedback scheme.

When building the toolkit, it was important to make the interface as extendable

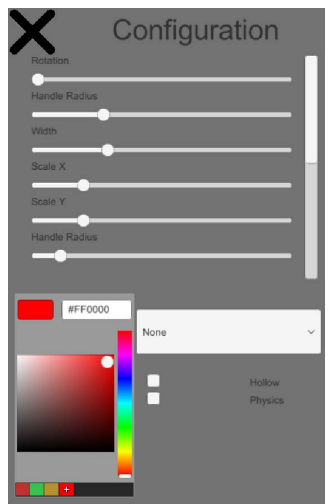

Figure 4: Run-time configuration menu. 
as possible. This way, the

toolkit would not impose constraints on design or otherwise be an obstacle to design completion. The toolkit allows extensibility through the ability to have user-defined functions execute as the slider percentage changes. This eliminates the need for a designer to manually change the slider's source to implement custom behaviour or constantly poll a slider value. Thus, this can greatly reduce the overhead for custom slider behaviour customization.

\section{Usage Data}

In both HCI and ACI, the collection of usage data is important to understand how an interface is used. This is even more important in ACI as this is the only way that interface effectiveness can be measured. In this toolkit, usage data is collected with a focus on touch events as they have been useful in the past [14].

Each touch interaction is collected into a vector. The system logs when a touch event starts, the path it follows, and when it ends. All touch events are logged, meaning multitouch interactions are also logged. The path points are collected at a fixed sample rate and not at each frame. This is done to avoid a variable frame rate from weighing the data incorrectly. The touch events collected by the system can then be analyzed by the designer.

In addition to touch events, trial data is logged. A trial here is defined as the configuration and events collected between the start of the slider handle movement and its completion or termination. With the high amount of configurability at run-time, manually keeping track of all configurations could be difficult. As such, the slider configuration is encoded and saved for each trial. Each trial log includes the start and end-time. A trial is automatically marked terminated when the slider enters the end state, but can also be manually terminated. If the end state is reached the trial is marked as a success otherwise it is marked as a failure.

\section{DISCUSSION AND CONCLUSION}

The toolkit that we have created can significantly reduce the interface iteration overhead when designing gestural interfaces for animal users. We accomplish this by providing designers with tools to quickly prototype and obtain usage metrics for analysis.

Similar to existing interface design tools, this toolkit hides the complexities of implementation from the designer and instead provides functionality and variables through a simple programming interface. By doing this, creating an animalfocused interface is simplified greatly. The toolkit also encourages expansion through exposing events and variables of the slider, allowing any number of novel interactions to be built upon it.
In addition to making an interface easy to build, it reduces the overhead of iterative changes. Determining animal specific aesthetic choices for interfaces is time-consuming, especially when working with a species for the first time. Here, we allow aesthetic variables to be modified during run-time to decrease the time between aesthetic iteration and provide more opportunities for the animal to be a design participant.

Animal design participation is an important part of this toolkit. By involving the animal in the design, we are more likely to make interfaces that enrich the animal instead of exploiting it. Analyzing qualitative data about the interaction between the animal and the interface can expose issues in the design that are not apparent from observation. The toolkit collects usage data that has successfully been used in the past to determine design errors. In this way, the system makes animal-centric design much easier to practice.

\section{Future Work}

The toolkit only allows an interface with a single type of interactive element. In the future, we hope to implement other familiar interface elements into the toolkit with the same level of configurability. By doing so, the task of making a full animal interface will be easily achievable. Additionally, the toolkit only provides the designer with raw metric data without much context other than the time at which it was collected. The toolkit will be expanded to display the usage data in a more informative and actionable way.

\section{ACKNOWLEDGEMENTS}

We would like to thank Ceara Byrne and Larry Freil for the insights given over the course of this project. We would also like to thank the GVU Center for providing us with the facilities to carry out ACI studies.

The work presented here was completed under National Science Foundation NSF Grant IIS-1525937.

\section{REFERENCES}

[1] Ulrike Aust, Friederike Range, Michael Steurer, and Ludwig Huber. 2008. Inferential reasoning by exclusion in pigeons, dogs, and humans. Animal Cognition 11, 4 (2008), 587-597. https://doi.org/10. 1007/s10071-008-0149-0

[2] Alethea Blackler and Jörn Hurtienne. 2007. Towards a unified view of intuitive interaction: definitions, models and tools across the world. Kindsmüller, M. C. \& Mahlke, S. (Hrsg.), MMI Interaktiv - User Experience: 1, 13 (2007), 36-54. https://doi.org/10.1016/j.coph.2013.08. 013S1471-4892(13)00159-8[pii]

[3] Alethea Blackler, Vesna Popovic, and Doug Mahar. 2010. Investigating users' intuitive interaction with complex artefacts. Applied Ergonomics 41, 1 (jan 2010), 72-92. https://doi.org/10.1016/J.APERGO. 2009.04.010

[4] Ceara Byrne, Ryan Kerwin, Jay Zuerndorfer, Scott Gilliland, Zehua Guo, Melody Jackson, Thad E Starner, and Georgia Tech. 2014. TwoWay Communication between Working Dogs and Their Handlers. IEEE Pervasive Computing 13, 2 (2014), 80-83. https://doi.org/10.1109/ MPRV.2014.38 
[5] Melody Y. Ivory and Marti A. Hearst. 2001. The state of the art in automating usability evaluation of user interfaces. Comput. Surveys 33, 4 (dec 2001), 470-516. https://doi.org/10.1145/503112.503114

[6] MJ Michael J. Muller and Sarah Kuhn. 1993. Participatory design. Commun. ACM 36, 6 (1993), 24-28. https://doi.org/10.1145/153571. 255960

[7] Irene M Pepperberg. 1983. Cognition in the African Grey parrot: Preliminary evidence for auditory/vocal comprehension of the class concept. Animal Learning \& Behavior 11, 2 (jun 1983), 179-185. https: //doi.org/10.3758/BF03199646

[8] Bonnie M Perdue, Ã Andrea W Clay, Diann E Gaalema, Terry L Maple, Tara S Stoinski, Zoo Atlanta, Palm Beach Zoo, and West Palm Beach. 2012. Technology at the Zoo : The Influence of a Touchscreen Computer on Orangutans and Zoo Visitors. 39, October 2010 (2012), 27-39. https://doi.org/10.1002/zoo.20378

[9] Patricia Pons, Javier Jaen, and Alejandro Catala. 2017. Towards Future Interactive Intelligent Systems for Animals. In Proceedings of the 22nd International Conference on Intelligent User Interfaces - IUI '17. ACM Press, New York, New York, USA, 389-400. https://doi.org/10.1145/ 3025171.3025175

[10] Friederike Range, Ulrike Aust, Michael Steurer, and Ludwig Huber 2008. Visual categorization of natural stimuli by domestic dogs. Animal Cognition 11, 2 (2008), 339-347. https://doi.org/10.1007/ s10071-007-0123-2

[11] Jennifer Vonk, Stephanie E. Jett, and Kelly W. Mosteller. 2012. Concept formation in American black bears, Ursus americanus. Animal Behaviour 84, 4 (oct 2012), 953-964. https://doi.org/10.1016/j.anbehav. 2012.07.020

[12] Lisa J. Wallis, Friederike Range, Enikő Kubinyi, Durga Chapagain, Jessica Serra, and Ludwig Huber. 2017. Utilising dog-computer interactions to provide mental stimulation in dogs especially during ageing. Proceedings of the Fourth International Conference on AnimalComputer Interaction - ACI2017 (2017), 1-12. https://doi.org/10.1145/ 3152130.3152146

[13] Daniel Wigdor and Dennis Wixon. 2011. Brave NUI World. Elsevier. https://doi.org/10.1016/C2009-0-64091-5

[14] Clint Zeagler, Ceara Byrne, Giancarlo Valentin, Larry Freil, Eric Kidder, James Crouch, Thad Starner, and Melody Moore Jackson. 2016. Search and rescue: dog and handler collaboration through wearable and mobile interfaces. Proceedings of the Third International Conference on Animal-Computer Interaction - ACI '16 (2016), 1-9. https: //doi.org/10.1145/2995257.2995390

[15] Clint Zeagler, Scott Gilliland, Larry Freil, Thad Starner, and Melody Jackson. 2014. Going to the dogs: towards an interactive touchscreen interface for working dogs. Proceedings of the 27th annual ACM symposium on User interface software and technology (2014), 497-507. https://doi.org/10.1145/2642918.2647364

[16] Clint Zeagler, Jay Zuerndorfer, Andrea Lau, Larry Freil, Scott Gilliland, Thad Starner, and Melody Moore Jackson. 2016. Canine computer interaction: towards designing a touchscreen interface for working dogs. Proceedings of the Third International Conference on Animal-Computer Interaction - ACI '16 (2016), 1-5. https://doi.org/10. 1145/2995257.2995384 